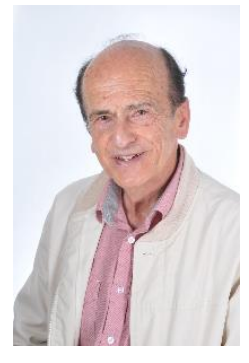

\author{
Professor Mieczyslaw Pokorski \\ Faculty of Health Sciences \\ Opole University, Opole \\ Poland
}

\title{
JSPS - PAN Webinar - November 2020
}

It takes me great pleasure to confirm the materialization of the efforts to organize a joint research seminar on medical rehabilitation under the aegis of the Japan Society for the Promotion of Science (JSPS) and the Polish Academy of Sciences (PAN). Due to the spiking second wave of COVI-19 infections, the event will be held on the Tokyo-based ZOOM platform on November 1214,2020 . The meeting will consist of presentations, lectures, discussions, and a practice workshop. A multisystem inflammatory nature of COVID-19 disease has created a spate of difficult to overcome challenges. Beside threatening acute respiratory distress syndrome, neurologic symptoms are a frequent accompaniment involving both and peripheral nervous systems. The disease is a scourge that increases the need for effective post-infection rehabilitation in both pulmonary and neuromuscular realms, burdening the already stretched medical rehabilitation, healthcare, and socioeconomic resources. In this context, a rehabilitative paradigm shift from the manual therapist-assisted to 'motivated exercise' advocated by the Takizawa method developed at the International Biophilia Academy (IBRA) in Tokyo comes in even handier.

Motivation is an indispensable feature in education, learning, and overall progress in activities somebody desires to perform. Likewise, it appears that 'brain's self-motivation' to reestablish and synchronized disordered transmission in neural pathways and optimally use functional and cognitive reserves is essential in physiotherapy, particularly in neurologic handicaps but also as the antiaging strategy. Additionally, the philosophy of 'motivated exercise' undauntingly promogulated by the Takizawa method takes a major burden off therapists who are in increasingly shorter supply in the face of longevity and the development of age-related health problems. This webinar meeting is going to report some of the recent high-quality data in the neuromuscular rehabilitative space pointing to significant motion and return-of-movement benefits. Besides the updated knowledge on recent achievements in 'motivated-exercise', technical innovations for myopathic and neuropathic physiotherapy and pulmonary post-COVID-19 physiotherapy will be addressed.

Tailored physiotherapy choices should be developed to meet the needs of individual patients and put the self-motivation training at the center stage. The sharing of scientific knowledge is key to disseminate progress in 'motivated exercise' and help increase the effectiveness of rehabilitation. The JSPS-PAN webinar will hopefully be a practice-changer in recuperationmodifying rehabilitation of neuromuscular disorders. 